\title{
A Ray Tracing Algorithm for Intelligent Transport Systems in Tunnels
}

\author{
Mingming Gan ${ }^{1}$, Zhinan $\mathrm{Xu}^{1}$, Veronika Shivaldova ${ }^{2}$, Alexander Paier ${ }^{3}$, Fredrik Tufvesson ${ }^{4}$, Thomas Zemen ${ }^{1}$ \\ ${ }^{1}$ FTW Forschungszentrum Telekommunikation Wien, Vienna, Austria \\ ${ }^{2}$ Institute of Telecommunications, Vienna University of Technology, Vienna, Austria \\ ${ }^{3}$ Kapsch TrafficCom AG, Vienna, Austria \\ ${ }^{4}$ Department of Electrical and Information Technology, Lund University, Lund, Sweden
}

\begin{abstract}
It is well-known that the radio wave propagation mechanisms inside a tunnel are different from the typical outdoor and indoor situations. Since the tunnels represent a significant type of vehicular environments, understanding the channel characteristics for the in-tunnel scenario is crucial for intelligent transport systems design. A widely used tool for simulating channel characteristics for outdoor and indoor scenarios is a deterministic propagation prediction tool, known as ray tracing (RT). However, RT applied for tunnel scenarios has not been studied adequately.

In this paper, we first evaluate the real-world in-tunnel vehicleto-vehicle radio channel measurements on the basis of timevarying power delay profile analysis. Secondly we introduce a RT tool that includes influence of the moving objects, to predict wave propagation mechanisms in the tunnel. In order to reduce computational complexity of RT, we suggest to combine an approximate algorithm for the higher-order reflection components with conventional RT and use a novel subdivision algorithm for modeling the diffuse scattering. Combining the higher-order reflection algorithm with conventional $\mathrm{RT}$ allows us to obtain more accurate delay spread results. The numerical simulations show that contribution of both the higher-order reflection and the diffuse components are equally important for the in-tunnel scenarios.
\end{abstract}

Keywords-ray tracing (RT), intelligent transport systems, vehicle-to-vehicle, tunnel, computational complexity.

\section{INTRODUCTION}

The performance and efficiency of intelligent transport systems (ITS) are mainly dependent on wireless communication systems. Vehicle-to-vehicle (V2V) wireless communications have received a lot of interest in recent years. The development of suitable communication standards requires the adequate understanding of the $\mathrm{V} 2 \mathrm{~V}$ propagation channels. It is known that the in-tunnel wave propagation characteristics are different from those in outdoor environments. Full comprehension of the in-tunnel wave propagation is of great importance for ITS to improve the safety in tunnel scenarios [1]. Moreover, it is obvious that the reliability of the radio-link is strongly dependent on propagation mechanisms. Therefore, effective channel prediction tools and models are required for the development of suitable communication systems and standards.

Deterministic modeling of electromagnetic wave propagation by means of ray tracing (RT) has been extensively used to simulate indoor and outdoor channel characteristics. It is used to trace all optical rays between the transmitter
(Tx) and receiver ( $\mathrm{Rx})$ based on the geometrical theory of propagation [2]. Up to now, RT includes not only specular components, such as reflection, penetration, and diffraction, but also diffuse scattering components. The accuracy, fed by a precise description of the propagation environment, is achieved at high computational complexity. There are only a few published studies of the RT tool in real tunnel environment in which simplified RT algorithm, without considering the detailed objects in the tunnel, have been used to study the behavior of radio wave propagation in empty tunnels. For example, RT simulations in [3] consider only the direct and reflection paths, where as in [4] the authors consider only the direct and specular components.

In this paper, we propose a RT algorithm for tunnel scenarios. The contributions of this paper are three-fold: i) The algorithm involves an effective subdivision algorithm for diffuse scattering [5] and an approximate higher-order reflection algorithm. ii) The accuracy of the presented algorithm is verified by comparing the time-varying power delay profile (PDP) and the time-varying delay spread with empirical measurements. iii) We analyze the composition of the timevarying PDP of the proposed tunnel RT algorithm.

The paper is organized as follows: Section II gives a short description of the tunnel scenario and empirical time-varying PDP results. Section III describes the tunnel RT algorithm, which includes conventional RT and a higher-order reflection algorithm. Simulation results and a discussion of the tunnel RT algorithm are presented in Section IV. Finally, we conclude in Section V.

\section{In-TunNEL MEASUREMEnTS AND TIME-VARYing ANALYSIS}

\section{A. In-Tunnel Measurements}

The measurements used in the present work were collected in the DRIVEWAY'09 measurement campaign [6] in the Øresund tunnel connecting Denmark and Sweden. The measurements are started when the Tx is inside the tunnel and the Rx enters the tunnel at $t=2 \mathrm{~s}$, see Fig. 2. The channel impulse response $h(t, \tau)$ is measured over a time interval of $T=10 \mathrm{~s}$, with a time resolution of $t_{\mathrm{s}}=307.2 \mu \mathrm{s}$. Therefore, there are $N=32000$ snapshots in total. The measurement bandwidth is $B=240 \mathrm{MHz}$ at a carrier frequency of $f_{\mathrm{c}}=5.6 \mathrm{GHz}$. The distance between vehicles is about $d=120 \mathrm{~m}$ and their 
speed is kept constant at $v_{\mathrm{Rx}}=v_{\mathrm{Tx}}=105 \mathrm{~km} / \mathrm{h}$ for the whole time interval. Both the Tx- and $\mathrm{Rx}$ - vehicle are equipped with a linear antenna array consisting of 4 elements each, so that there are $L=16$ channel impulse responses. Moreover, each element is mainly radiating in one of the four directions: left, right, back and front, thus the antenna array could cover $360^{\circ}$ in the azimuth plane. The picture in Fig. 1 is taken from the video of the measurements when both, Tx and $\mathrm{Rx}$, are inside the tunnel.

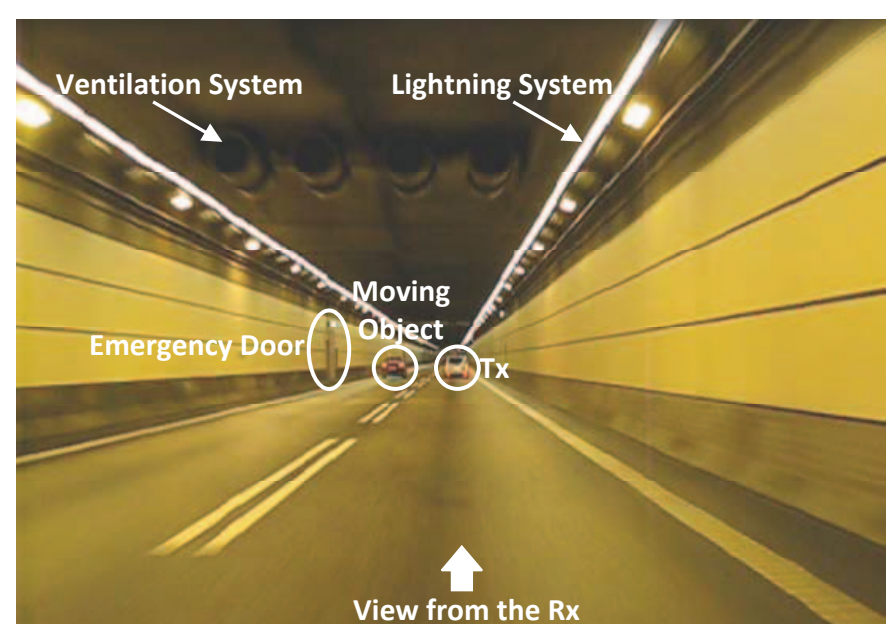

Fig. 1. Video snapshot representing measurement environment.

\section{B. Analysis of Measured Time-Varying PDP}

The observed fading process of the $\mathrm{V} 2 \mathrm{~V}$ channel is nonstationary [6]. The local scattering function (LSF) is a useful quantity for characterizing non-stationary time-variant channels, which is a short-term representation of the power spectrum in the observed fading process [1]. The time-varying PDP, shown in Fig. 2, can be calculated by projecting the LSF in the delay domain [7]. There are $N_{\mathrm{tv}}=250$ time stamps over

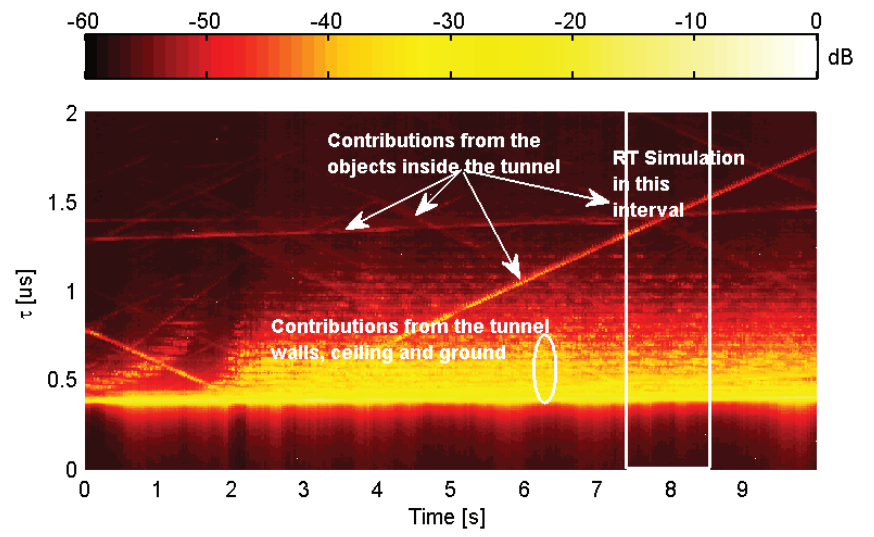

Fig. 2. Time-varying PDP measured over interval of $T=10 \mathrm{~s}$.

time interval $T=10 \mathrm{~s}$ for the time-varying PDP analysis. Multiple signal components parallel to the direct line-of-sight
(LOS) component are present in Fig. 2, which are mainly caused by the reflections from the tunnel walls, its ceiling and floor. Besides these significant contributions, the components from objects inside the tunnel are also obvious. Due to the high computational complexity of RT, we limit the simulation of the channel impulse response to $1 \mathrm{~s}$, corresponding to $t=7.5 \mathrm{~s}, \ldots, 8.5 \mathrm{~s}$, in the following analysis.

\section{TUNNEL RT ALGORITHM}

\section{A. Conventional RT}

A conventional RT tool typically uses three-dimensional (3D) model considering three major wave propagation mechanisms: (i) line of sight (LOS), (ii) specular contributions and (iii) diffuse scattering. The specular contributions include reflection, penetration and diffraction components. All propagation paths connecting $\mathrm{Tx}$ and $\mathrm{Rx}$ are calculated based on the geometrical and electromagnetic description of the environment and the relevant propagation mechanisms. The geometrical relationships between the incident and the reflected/penetrated/diffracted rays are based on optical principles [8]. Complex dyadic coefficients for the reflection and penetration are obtained using Fresnel formulas, while the diffraction coefficient is calculated by the uniform theory of diffraction (UTD) [9]. RT enables the electric field calculation in amplitude, phase and polarization at the Rx position. It should be mentioned that our RT tool is implemented in Matlab and it has been accelerated by using MEX functions.

Among all the propagation paths, the number of diffuse scattering paths takes the largest proportion, which influences the computational complexity directly. A directive pattern model, assuming that the scattering lobe is steered toward the direction of the specular reflection, is used to model the contribution of the diffuse part of the channel [10]. A rough surface is divided into multiple scattering tiles, firstly. The typical method used for evaluating the tile size is recursively dividing the surface until the far-field condition is satisfied [11]. In order to save simulation time, a new subdivision algorithm is presented in [5]. This algorithm depends on concentric circles, which assumes that diffuse scattering tiles are equally sized circular segments within the same radius $\Delta d$ on a certain surface and the relevant path originates from the center of each tile. The proper tile size

$$
d S=\pi \cdot \Delta d^{2}=\pi \cdot\left(\frac{c}{2 \cdot B}\right)^{2},
$$

is defined according to the system bandwidth $B$, where $c$ is the speed of light. It is known that the delay resolution is $\Delta \tau=1 / B$. This method significantly reduces the computational complexity of RT with no loss in accuracy [5].

\section{B. Approximate Higher-Order Reflection Algorithm}

In conventional RT, the calculation of reflection paths consists of two main steps: i) the visibility procedure and ii) the back track procedure. The visibility procedure is used to build up the image tree and the back track procedure helps to determine the real reflection paths. However, the required 
storage space for saving the information of the image tree increases exponentially with the reflection order. Our RT tool has solved this storage issue by saving only the information related to the real reflection paths in the image tree. Despite of this, the computational complexity, involved in the back track procedure, is tremendously increased referring to the higherorder reflection case. Therefore, an approximate higher-order reflection algorithm is considered to be combined with the conventional RT algorithm.

This algorithm does not consider the objects inside the tunnel, so that the tunnel is treated as an equivalent rectangle [12]. The input for the algorithm includes the positions of Tx and $\mathrm{Rx}$, the width $2 a$ and the height $2 b$ of the tunnel, as well as the permittivity and conductivity of the vertical and horizontal walls. The set of reflection images of the Tx on the excitation plane are shown in Fig. 3. The origin of the coordinate system is located at the center of the rectangular tunnel. The propagation paths related to image $I_{p, q}$ experiences $|p|$ times vertical reflections and $|q|$ times horizontal reflections. Regardless of the reflection order, the incidence angles on the vertical and horizontal walls, $\beta_{v}$ and $\alpha_{h}$, remain the same for a certain path.

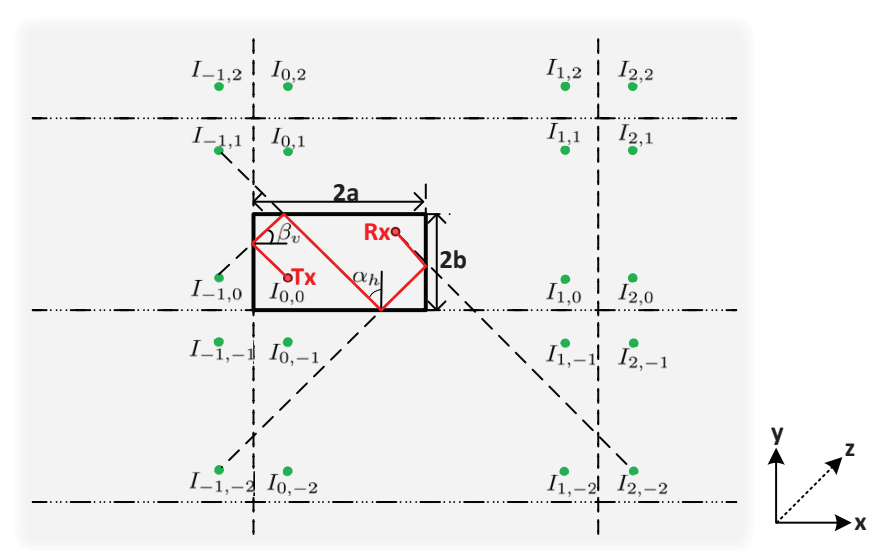

Fig. 3. The set of images within the tunnel.

For a Tx positioned at $\left(x_{0}, y_{0}, z_{0}\right)$, the coordinate $\left(x_{\mathrm{i}}, y_{\mathrm{i}}, z_{\mathrm{i}}\right)$ of the image point $I_{p, q}$ can be calculated as

$$
\left\{\begin{array}{l}
x_{\mathrm{i}}=2 a \cdot p \pm x_{0} \\
y_{\mathrm{i}}=2 b \cdot q \pm y_{0} \\
z_{\mathrm{i}}=z_{0}
\end{array}\right.
$$

where the positive sign is selected if $p$ or $q$ is even while the negative sign is selected if $p$ or $q$ is odd. The Tx and Rx antennas used for the RT simulation are vertical half-wave dipole antennas in the present work. The electric field for a single order reflection component, can be expressed as

$$
E_{\mathrm{r}}=\left[\bar{g}_{\mathrm{r}}^{\mathrm{R}}\right]^{*} \cdot \overline{\overline{\mathbf{T}}} \cdot \bar{g}_{\mathrm{r}}^{\mathrm{E}} \frac{e^{-j k r_{p, q}}}{r_{p, q}} E_{0},
$$

where $\bar{g}_{\{\cdot\}}^{\mathrm{E}}=\bar{g}_{\{\cdot\}}^{\mathrm{E}}\left(f_{\mathrm{c}}, \theta_{\mathrm{E}}, \phi_{\mathrm{E}}\right)$ and $\bar{g}_{\{\cdot\}}^{\mathrm{R}}=\bar{g}_{\{\cdot\}}^{\mathrm{R}}\left(f_{\mathrm{c}}, \theta_{\mathrm{R}}, \phi_{\mathrm{R}}\right)$ are the complex vectors accounting for the $\mathrm{TX} / \mathrm{Rx}$ antenna polarization and amplitude gains in the direction of the propagation wave, $\theta_{\{\cdot\}}$ and $\phi_{\{\cdot\}}$ indicate the azimuth and elevation directions of the transmitted/received wave, $\{\cdot\}^{*}$ designates the complex conjugate, $\overline{\overline{\mathbf{T}}}=R_{\mathrm{v}}^{|p|} \cdot R_{\mathrm{h}}^{|q|}$ is the reflection coefficient, $R_{\mathrm{v}}$ and $R_{\mathrm{h}}$ are the vertical and horizontal polarization coefficients [13], $k$ is the wave number, $r_{p, q}$ is the distance between the image point $I_{p, q}$ and $\mathrm{Rx}$, which is equal to the total length of the propagation path between $\mathrm{Tx}$ and $\mathrm{Rx}$, and $E_{0}$ is the emitted field. It is worth mentioning that the corresponding $\phi_{\{\cdot\}}$, determining the half-wave dipole antennas radiation pattern, can be easily obtained.

Compared to the reflection algorithm in conventional RT, the approximate higher-order reflection algorithm requires no back track procedure. Based on the visibility procedure, all relevant information for the path calculation can be easily obtained. Therefore, we propose a hybrid approach by combining this higher-order reflection algorithm with the conventional RT to predict the propagation channel in real tunnels.

\section{NUMERICAL RESULTS}

\section{A. RT Setup}

A 3-D view of the tunnel scenario used for RT is illustrated in Fig. 4, where the different materials are sketched with different colors. It should be noted that we do not have the

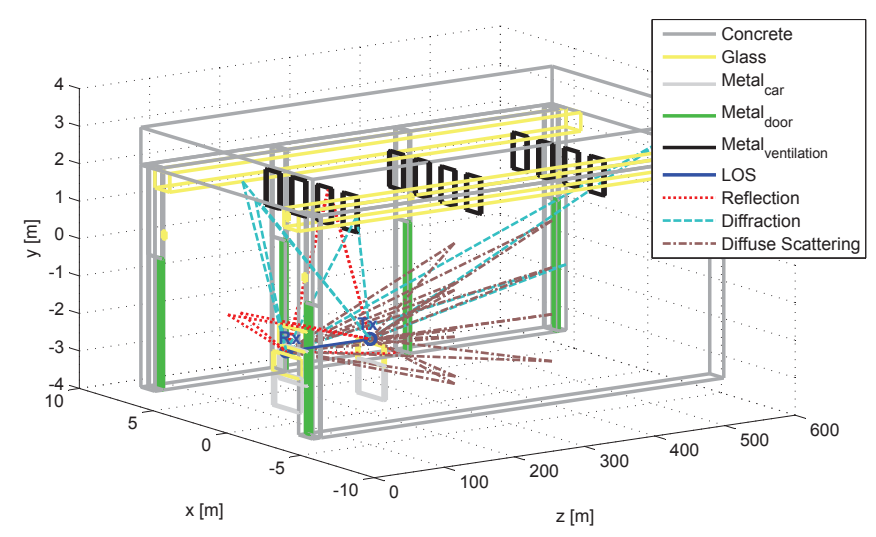

Fig. 4. A 3-D view of the tunnel scenario.

detailed description of the tunnel. The dimensions of the tunnel and the objects inside are found in [14] and in the video of the measurements. Each block is modeled as a rectangular solid and the moving object is also included by our RT tool. The width is $2 a=11.65 \mathrm{~m}$ and the height is $2 b=6.93 \mathrm{~m}$, while the considered length is $586 \mathrm{~m}$. It should be noted that the length of the tunnel is longer than the considered length. The reason is that the propagation paths from the deeper inside of the tunnel, most likely came from the diffuse scattering, would contain low power, but it would result in extremely high computational complexity. The dielectric properties are included in the input database of RT, in which the metallic block is assumed as a perfect electric conductor. The values of relative permittivity $\varepsilon_{r}$ and the conductivity $\sigma$ of the materials are: $\varepsilon_{r, \mathbf{c}}=8.92$ and $\sigma_{\mathbf{c}}=0.046 \mathrm{~S} / \mathrm{m}$ for concrete blocks, and 
$\varepsilon_{r, \mathbf{g}}=3.7$ and $\sigma_{\mathbf{g}}=0 \mathrm{~S} / \mathrm{m}$ for glass blocks [15]. According to the simulation parameters, $d S$ is $1.23 \mathrm{~m}^{2}$ for the proposed subdivision algorithm based on (1). The Tx and Rx antennas used for the RT simulation are half-wave dipole antennas. Conventional RT takes into account the following propagation mechanisms: LOS, reflection (up to the fourth order), single diffraction, single bounce scattering, scattering-reflection and reflection scattering cases. Penetration has been embedded into all other mechanisms. Some propagation paths are visualized in Fig. 4. For the approximate higher-order reflection paths, the vertical reflection order $|p|$ and horizontal reflection order $|q|$ satisfy the relationship as $4 \leq|p|+|q| \leq 40$. We assume that components with reflection order larger than 40 can be neglected as shown in [3]. In order to make the simulation results comparable to the measurements, RT performs at $N=3200$ snapshots in the time interval $T=1 \mathrm{~s}$. The simulation time of the tunnel RT for one snapshot is about $407 \mathrm{~s}(2.4 \mathrm{GHz}$ Intel Core i7 CPU with 8GB RAM). Furthermore, $N_{\mathrm{tv}}=25$ time stamps over the time interval $T=1 \mathrm{~s}$ is used for the timevarying PDP analysis.

\section{B. Analysis of Modeled Time-Varying PDP}

The normalized time-varying PDP $\hat{P}(t, \tau)$ of the tunnel RT algorithm, where $t$ indicates the time and $\tau$ indicates the delay, is shown in Fig. 5. The contributions from the objects inside the tunnel can be observed clearly. To clarify, we focus here at

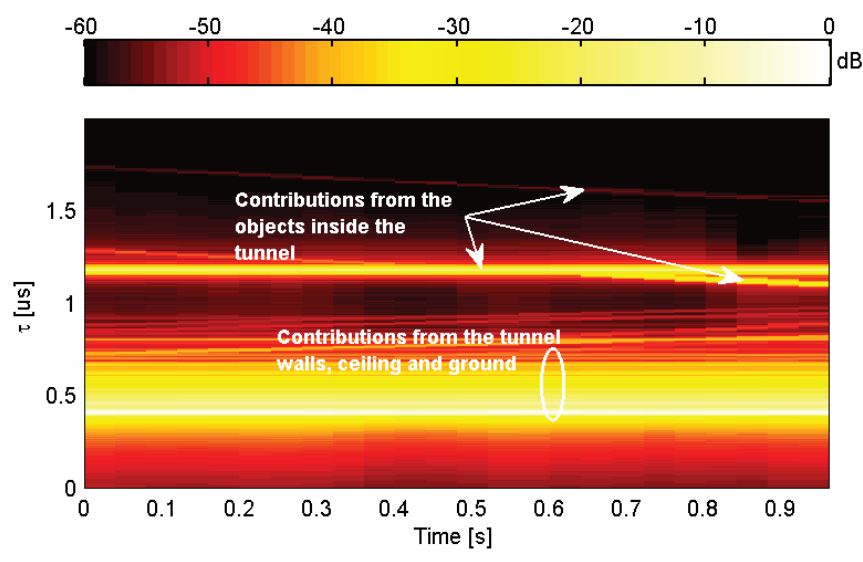

Fig. 5. Time-varying PDP simulated with the tunnel RT algorithm for time interval of $T=1 \mathrm{~s}$.

one instant and compare the time-varying PDPs at time stamp $n_{\mathrm{tv}}=20$, where $n_{\mathrm{tv}} \in\left\{0, \ldots, N_{\mathrm{tv}}-1\right\}$ denotes the time stamp index. The results are shown in Fig. 6. From Fig. 6 (a), it can be seen that the normalized PDPs based on measurements and the tunnel RT algorithm are comparable to each other. The peaks appearing between $1 \mu \mathrm{s}$ and $1.5 \mu \mathrm{s}$, marked by the red arrows, are mainly caused by the moving object and the ventilation system. The shifts in the delay domain and the imprecision of the power between the measurements and the tunnel RT are unavoidable in the present work, because the environmental information to the input of RT is not accurate enough. In the lower plots Fig. 6 (b)-(d), we analyze the composition of the PDP of the tunnel RT algorithm. Due to the dimension of the tunnel, the considered fourth-order reflection contributions are not enough, shown in Fig. 6 (b), which is usually used for typical outdoor and indoor scenarios. In Fig. 6 (c), it can be observed that the higher-order reflection components play a more important role in the delay interval $0.45 \mu \mathrm{s}-0.75 \mu \mathrm{s}$, while the diffuse scattering components are more significant after $0.75 \mu \mathrm{s}$, shown in Fig. 6 (d). Moreover, it can be concluded that the diffuse scattering components contain more power when they are close to the specular components.

(a)
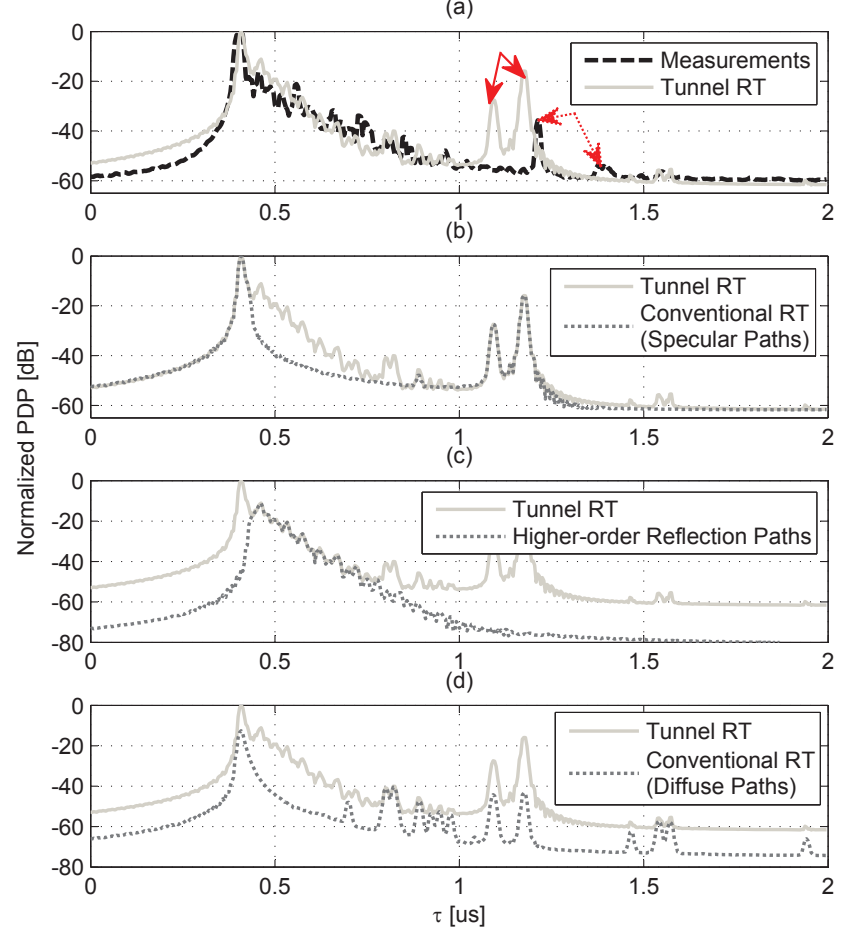

Fig. 6. Time-varying PDP based on the tunnel RT algorithm at time stamp $n_{\mathrm{tv}}=20$.

\section{Analysis of Time-Varying Delay Spread}

The time-varying delay spread at discrete time $n_{\mathrm{tv}}$ is defined as [16]

$$
\sigma_{\tau}\left(n_{\mathrm{tv}}\right)=\sqrt{\frac{\sum_{i=0}^{L-1}\left(i \tau_{\mathrm{s}}\right)^{2} \hat{P}\left(n_{\mathrm{tv}}, i\right)}{\sum_{i=\mathrm{o}}^{L-1} \hat{P}\left(n_{\mathrm{tv}}, i\right)}-\left(\frac{\sum_{i=\mathrm{o}}^{L-1}\left(i \tau_{\mathrm{s}}\right) \hat{P}\left(n_{\mathrm{tv}}, i\right)}{\sum_{i=\mathrm{o}}^{L-1} \hat{P}\left(n_{\mathrm{tv}}, i\right)}\right)^{2}},
$$

where $\hat{P}\left(n_{\mathrm{tv}}, i\right)$ is the sampled time-varying PDP and $i \in$ $\{0, \ldots, L-1\}$ denotes the delay index. We compare the delay spread of the measurements and different RT algorithms in Fig. 7. The delay spread $\sigma_{\tau}$ of the measurements oscillates around $0.1 \mu \mathrm{s}$. The tunnel RT algorithm provides a similar trend and mean value as the measurements. Conventional RT provides a slightly higher $\sigma_{\tau}$ value than the tunnel algorithm, while the 
higher-order reflection algorithm alone provides a constant $\sigma_{\tau}$ value of $0.02 \mu \mathrm{s}$. It should be noted that we use the higherorder reflection algorithm as a reference here, which considers the LOS and first-order to 40th-order reflection cases. Because the algorithm only depends on the width and height of the tunnel, and the Tx and Rx positions, the obtained propagation paths are the same at any time stamp when the environment is kept constant.

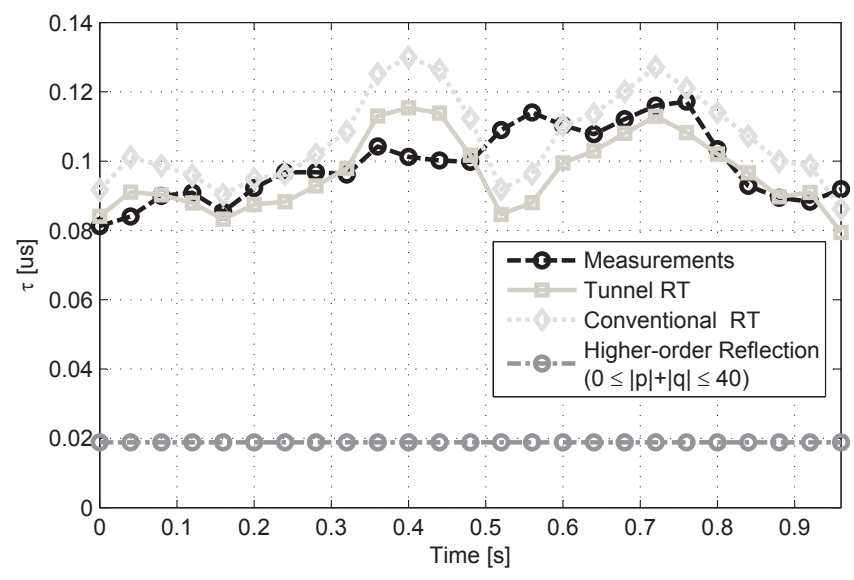

Fig. 7. Time-varying delay spread comparison.

\section{CONCLUSiON}

In this paper, we presented a RT algorithm for modeling vehicle-to-vehicle in-tunnel channels. It is well-known that implementation of the reflection and diffuse scattering algorithms in conventional RT results in extremely high computational complexity. Therefore, we propose to combine the conventional RT enhanced with an effective subdivision algorithm for diffuse scattering and the approximate higher-order reflection algorithm, to yield the novel RT algorithm for the intunnel environments. The accuracy of the proposed algorithm is verified by comparing its time-varying PDP and delay spread to the ones obtained from the real-world measurements. This comparison underlines high precision of our algorithm. Furthermore, we have shown that by implementing solely the higher-order reflection algorithm the important contributions from the objects inside the tunnel can not be distinguished. Consequently, the tunnel RT algorithm clearly outperforms the sole higher-order reflection algorithm with respect to the accuracy of the time-varying delay spread. Finally, the numerical evaluations have shown that in realistic tunnel environments the diffuse scattering components are as important as the higher-order reflection components.

\section{ACKNOWLEDGMENT}

The author would like to thank Miss Xuhong Li for assistance with speeding up the ray tracing simulation. This work was performed in the FTW project ITS Evolution, a scientific cooperation co-funded by ASFINAG, Kapsch TrafficCom AG, and Vienna University of Technology. The Austrian Competence Center FTW Forschungszentrum Telekommunikation Wien $\mathrm{GmbH}$ is funded within the program COMET - Competence Centers for Excellent Technologies by BMVIT, BMWFJ, and the City of Vienna. The COMET program is managed by the FFG.

\section{REFERENCES}

[1] L. Bernadó, A. Roma, A. Paier, T. Zemen, N. Czink, J. Karedal, A. Thiel, F. Tufvesson, A. Molisch, and C. Mecklenbräuker, "In-tunnel vehicular radio channel characterization," in Vehicular Technology Conference (VTC Spring), 2011 IEEE 73rd, May 2011, pp. 1-5.

[2] F. Fuschini and G. Falciasecca, "A mixed rays - modes approach to the propagation in real road and railway tunnels," Antennas and Propagation, IEEE Transactions on, vol. 60, no. 2, pp. 1095-1105, Feb. 2012.

[3] C. Zhou, J. Waynert, T. Plass, and R. Jacksha, "Modeling RF propagation in tunnels," in Antennas and Propagation Society International Symposium (APSURSI), 2013 IEEE, July 2013, pp. 1916-1917.

[4] G. Ching, K. Tsuda, and Y. Kishiki, "Analysis of path gain inside tunnels based on FDTD and ray tracing methods," in Electromagnetic Theory (EMTS), Proceedings of 2013 URSI International Symposium on, May 2013, pp. 644-647.

[5] M. Gan, X. Li, F. Tufvesson, and T. Zemen, "An effective subdivision algorithm for diffuse scattering of ray tracing," in XXXI General Assembly and Scientific Symposium of the International Union of Radio Science (URSI), Aug. 2014, submitted.

[6] A. Paier, L. Bernadó, J. Karedal, O. Klemp, and A. Kwoczek, “Overview of vehicle-to-vehicle radio channel measurements for collision avoidance applications," in Vehicular Technology Conference (VTC 2010-Spring), 2010 IEEE 71st, May 2010, pp. 1-5.

[7] L. Bernadó, T. Zemen, A. Paier, J. Karedal, and B. Fleury, "Parametrization of the local scattering function estimator for vehicular-to-vehicular channels," in Vehicular Technology Conference Fall (VTC 2009-Fall), 2009 IEEE 70th, Sep. 2009, pp. 1-5.

[8] M. Gan, F. Mani, F. Kaltenberger, C. Oestges, and T. Zemen, "A ray tracing algorithm using the discrete prolate spheroidal subspace," in IEEE International Conference on Communications (ICC), June 2013.

[9] C. Oestges, B. Clerckx, L. Raynaud, and D. Vanhoenacker-Janvier, "Deterministic channel modeling and performance simulation of microcellular wide-band communication systems," Vehicular Technology, IEEE Transactions on, vol. 51, no. 6, pp. 1422 - 1430, Nov. 2002.

[10] V. Degli-Esposti, F. Fuschini, E. M. Vitucci, and G. Falciasecca, "Measurement and modelling of scattering from buildings," Antennas and Propagation, IEEE Transactions on, vol. 55, no. 1, pp. 143 - 153, Jan. 2007.

[11] F. Mani, F. Quitin, and C. Oestges, "Accuracy of depolarization and delay spread predictions using advanced ray-based modeling in indoor scenarios," EURASIP Jounal in Wireless Commmunications and Networking, vol. 2011, p. 11, 2011.

[12] Z. Sun and I. Akyildiz, "Channel modeling and analysis for wireless networks in underground mines and road tunnels," Communications, IEEE Transactions on, vol. 58, no. 6, pp. 1758-1768, June 2010.

[13] C. Gentile, F. Valoit, and N. Moayeri, "A raytracing model for wireless propagation in tunnels with varying cross section," in Global Communications Conference (GLOBECOM), 2012 IEEE, Dec. 2012, pp. 50275032.

[14] C. Marshall, "The Øresund tunnel - making a success of design and build," in Tunneling and Underground Space Technolody, vol. 58, no. 3, 1999, pp. 355-365.

[15] M. Gan, P. Meissner, F. Mani, E. Leitinger, M. Fröhle, C. Oestges, K. Witrisal, and T. Zemen, "Calibration of indoor UWB sub-band divided ray tracing using multiobjective simulated annealing," in IEEE International Conference on Communications (ICC), June 2014, to be presented.

[16] L. Bernadó, T. Zemen, F. Tufvesson, A. Molisch, and C. Mecklenbräuker, "Delay and Doppler spreads of nonstationary vehicular channels for safety-relevant scenarios," in Vehicular Technology, IEEE Transactions on, vol. 63, no. 1, Jan 2014, pp. 82-93. 